\title{
Late Infantile Neuronal Ceroid Lipofuscinosis in a Filipino Child with Epilepsy and Progressive Neurodegeneration
}

\author{
Mary Anne D. Chiong ${ }^{1,2}$ and Benilda C. Sanchez-Gan² \\ ${ }^{1}$ Institute of Human Genetics, National Institutes of Health, University of the Philippines Manila \\ ${ }^{2}$ Department of Pediatrics, College of Medicine and Philippine General Hospital, University of the Philippines Manila
}

\begin{abstract}
The neuronal ceroid lipofuscinoses correspond to a group of disorders characterized by neurodegeneration and intracellular buildup of auto-flourescent lipopigment (ceroid lipofuscin). They are classified by age of onset into infantile, late infantile, juvenile and adult forms. Among these, the late infantile type is caused by mutations in tripeptidyl peptidase 1 (TPP1) gene and is characterized by age of onset between 2-4 years, seizures, early progressive cognitive impairment and visual loss.
\end{abstract}

Our patient is a 4-year-old girl who presented at 2 years and 10 months old with seizures followed by ataxia, regression of skills and eventual visual decline. TPP1 enzyme activity was below normal for age. This report aims to increase the awareness of physicians on the cluster of symptoms characteristic of this disorder which will help facilitate early diagnosis and prompt institution of appropriate management.

Key Words: Late infantile neuronal ceroid lipofuscinosis, Neuronal ceroid lipofuscinosis type 2, Tripeptidyl peptidase 1

\section{Introduction}

The neuronal ceroid lipofuscinosis (NCL) are a group of genetically heterogenous and clinically variable neurodegenerative conditions characterized by progressive decline in cognition and motor capabilities, visual failure, epilepsy and premature death. ${ }^{1,2}$ They are one of the most commonly diagnosed hereditary progressive neurodegenerative diseases with a prevalence of approximately 1.5 to 9 per million population. The incidence of NCL ranges in different countries from 1.3 to seven per 100,000 live births. ${ }^{3}$

Traditionally, NCLs were classified according to the age of onset: infantile (INCL; 6 months to 2 years), late infantile (LINCL; 2-4 years), juvenile (JNCL; 4-10 years) and adult (ANCL; >10 years) leading to the simple supposition that there are four genes responsible for NCL disease, CLN1, CLN2, CLN3 and CLN 4, respectively. ${ }^{1,2,4}$ Over the years,

Corresponding author: Mary Anne D. Chiong, MD

Institute of Human Genetics

National Institutes of Health

University of the Philippines Manila

623 Pedro Gil St., Ermita, Manila 1000 Philippines

Telephone: +6325261725 local 114

Email: mdchiong1@up.edu.ph genes carrying mutations that cause NCL have been discovered by a variety of experimental approaches and today, at least 14 genes are implicated. ${ }^{4}$ Most show autosomal recessive inheritance except for the adult onset form of the disease which may be inherited in an autosomal dominant manner. ${ }^{4}$

The pathophysiology involves lysosomal accumulation in many organs and tissues of pigments that fluoresce under ultraviolet microscope which contain proteins, lipids and metals. ${ }^{3,5}$ Under the electron microscope, the accumulated material takes different forms: granular osmiophilic deposits (GRODs), curvilinear profiles (CLP), fingerprint profiles (FPP), as well as rectilinear complex (RLC) or so called condensed forms. Even though these stored materials are not type specific, the ultrastructural appearance was previously used to diagnose the disease together with the clinical presentation. However, the recent availability of enzyme assays and identification of disease causing mutations for specific NCL proteins have enabled more rapid and reliable diagnosis of the different disease subtypes. ${ }^{5}$

We report a Filipino child presenting with progressive neurologic deterioration with corresponding deficiency in the activity of tripeptidyl peptidase 1 (TPP1) enzyme that is deficient in late infantile neuronal ceroid lipofuscinosis.

\section{Clinical report}

The patient at the time of examination was 4 years of age. She is the second child of healthy non-consanguineous Filipino parents. The pregnancy, delivery and the neonatal course were uneventful. At 2 years and 10 months old, she had 2 episodes of generalized tonic clonic seizures associated with fever. She had persistence of partial or generalized seizures occurring 2-3 times weekly lasting for few seconds even in the absence of fever which were fairly controlled with Valproic acid. The cranial MRI was unremarkable. Her initial electroencephalogram (EEG) showed background slowing specifically in the occipital area. Developmental progress was still within normal limits for age.

At 3 years and 6 months of age, she was noted to have involuntary tremors of the bilateral hands and feet. This was later followed by frequent falls due to myoclonic jerks. A repeat EEG at 4 years of age showed low to medium voltage 


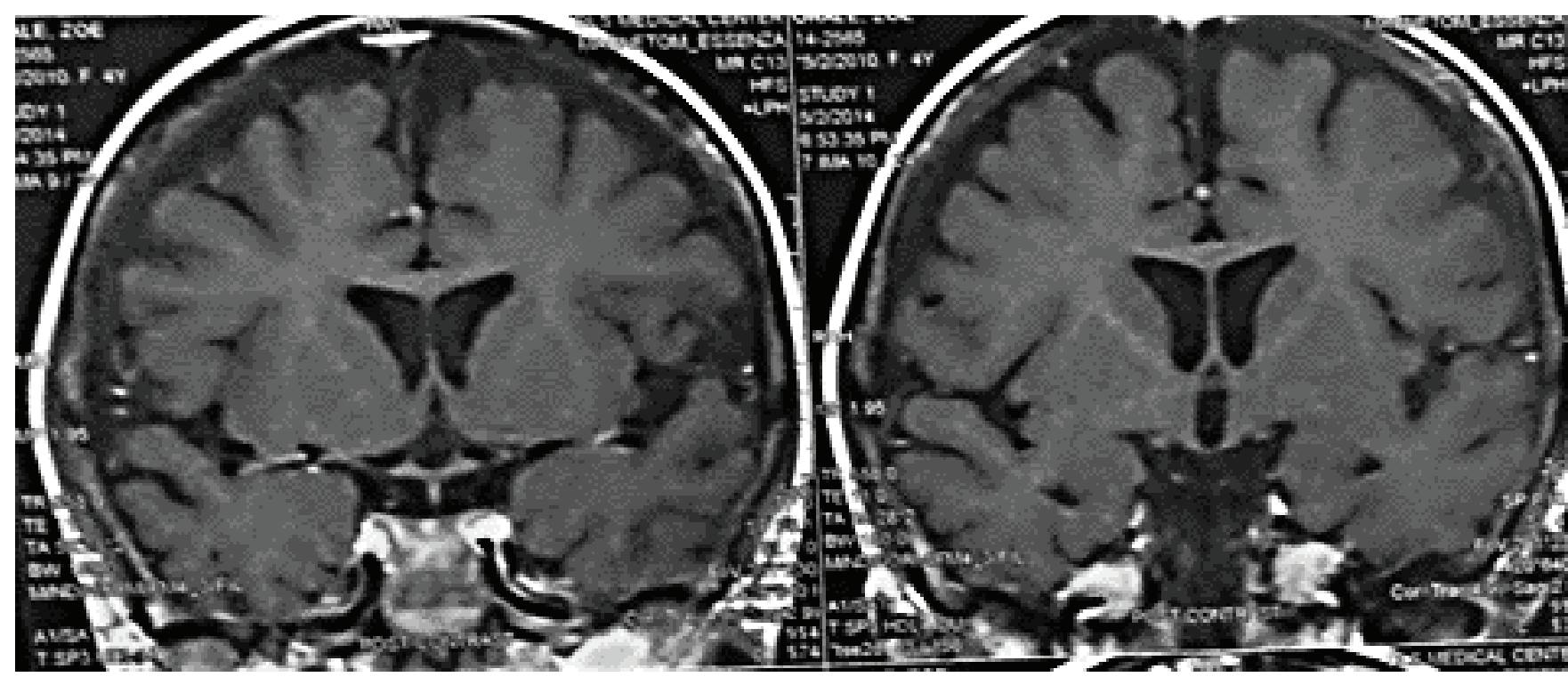

Figure 1. Coronal views of cranial MRI T1 weighted images at 4 years of age showing cerebro-cerebellar atrophy.

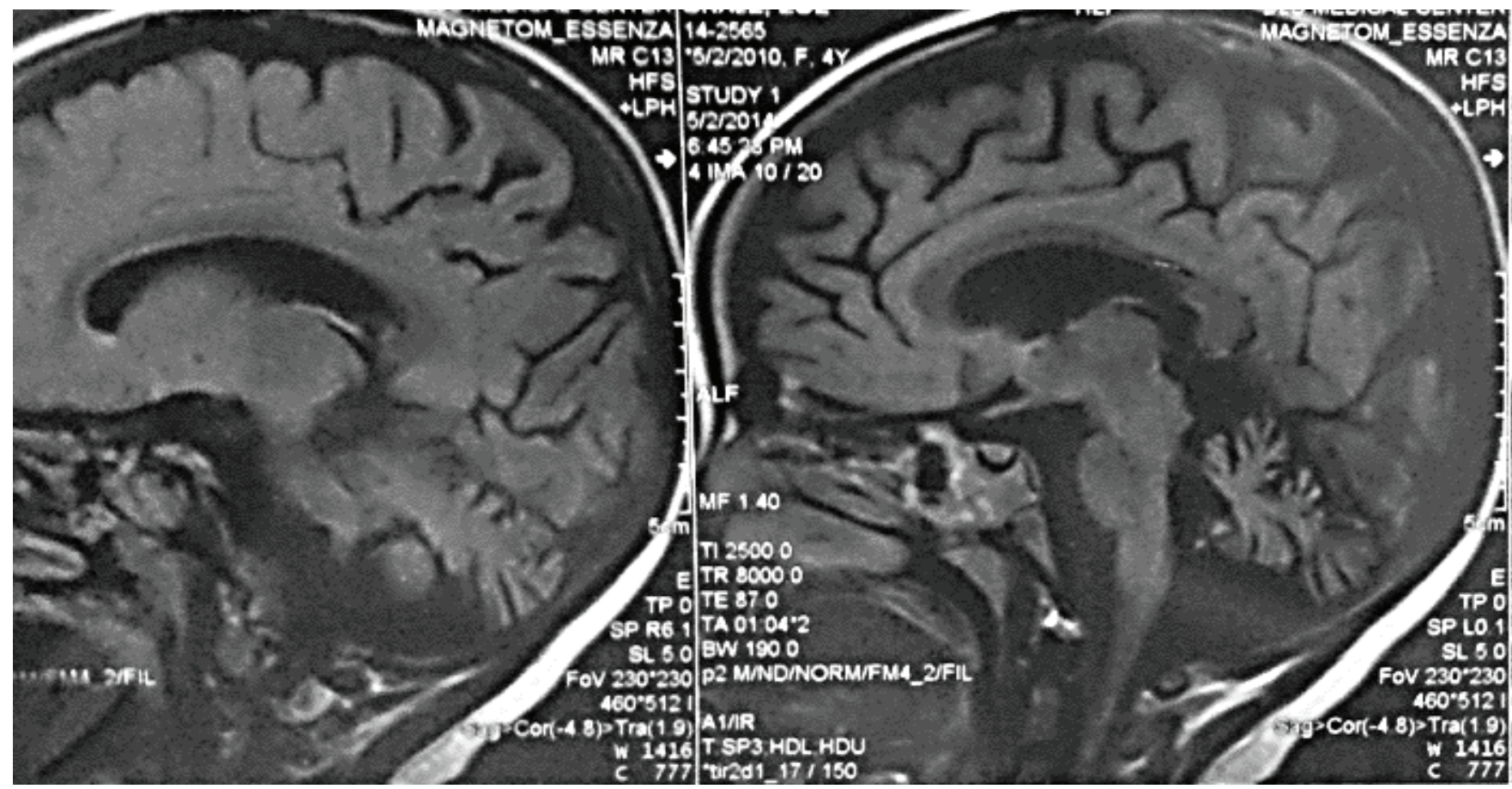

Figure 2. Sagittal views of cranial MRI T1 weighted images showing cerebro-cerebellar atrophy.

(3-4 $\mathrm{Hz}$ and $4-5 \mathrm{~Hz})$ activities seen diffusely, with occasional bursts of high voltage $1-2 \mathrm{~Hz}$ spike and slow waves on both occipital regions. A repeat cranial MRI revealed mild cerebro-cerebellar atrophy with few tiny flair hyperintense signals in the frontal lobe white matter (Figures 1, 2 and 3). Ophthalmologic examination showed that she was able to fixate well and follow moving objects. Fundoscopic examination showed clear media, optic disc was yellow orange, and with positive foveal reflex. However, error of refraction was noted. The baseline urine organic acid showed slight lactate elevation with no other significant metabolites. Plasma lactate was $1.2 \mathrm{mmol} / \mathrm{L}(0.4-2 \mathrm{mmol} / \mathrm{L})$. At around this time, despite the decrease in the frequency of the myoclonic seizures, she started to lose previously acquired skills such as counting numbers and identification of alphabets. Her expressive language skills did not progress and were now delayed for age. Her gait became ataxic. Neurologic examination showed a normal head circumference at 50th-85th percentile for age. There were brisk tendon reflexes in the lower extremities with a positive Babinski reflex. The grip was noted to be weak. 


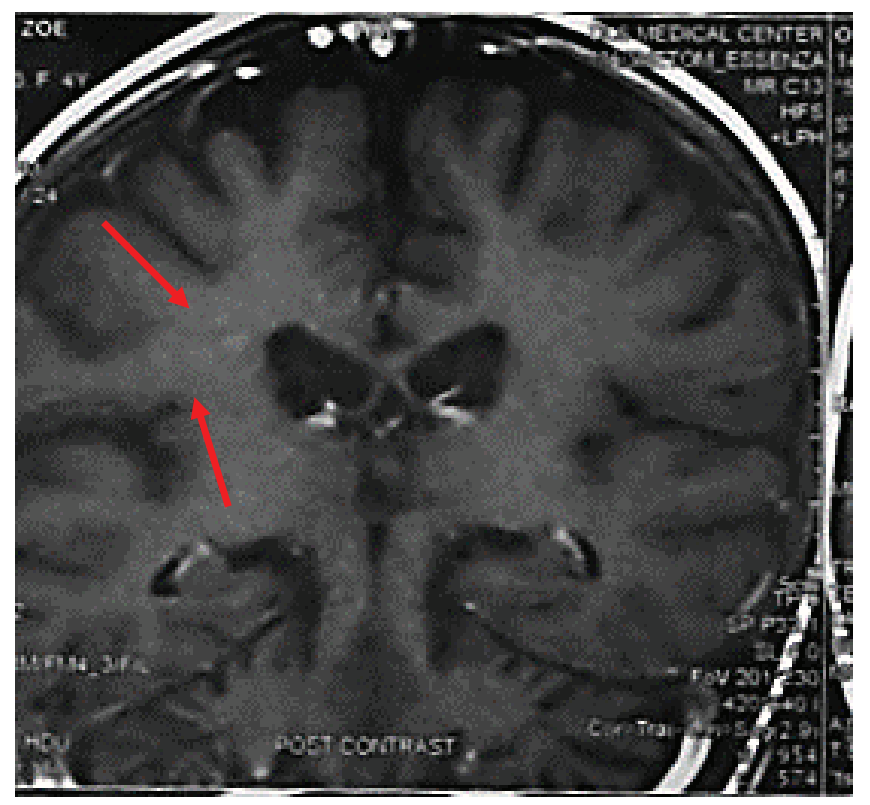

Figure 3. Coronal view, T1 weighted image showing hyperintense signals in the frontal lobe white matter.

Based on the MRI findings and the clinical features of seizures, ataxia and cognitive decline beginning past 2 years of age, neuronal ceroid lipofuscinosis, late infantile type was considered. Blood leukocytes were sent to the National Referral Laboratory for Peroxisomal, Lysosomal and Other Related Disorders in Adelaide, Australia. The results revealed a low activity of the enzyme Tripeptidyl peptidase 1 (TPP1) at $<0.1 \mathrm{nmol} / \mathrm{min} / \mathrm{mg}$ protein (NV 0.8-2.0). Mutation testing of the CLN2 gene causing late infantile neuronal ceroid lipofuscinosis was planned. Genetic counselling was provided to the family. Due to the condition being inherited in an autosomal recessive disorder, a $25 \%$ recurrence risk for succeeding pregnancies was quoted.

On follow up at 7 years of age, the patient's condition has progressed and she was already in a semi-vegetative state. She completely lost her ability to talk and walk. Eyesight has declined to near blindness. No formal ophthalmologic re-evaluation was done at this time. She has no difficulty in swallowing but could only tolerate pureed foods. The seizures, mainly myoclonic, occur infrequently and are well controlled by Leviteracetam and Valproic acid. The family has stopped providing rehabilitation therapy and only comfort care is being given to date.

\section{Discussion}

The clinical features, electrophysiologic, cranial imaging and biochemical results of this patient satisfied the diagnosis of late infantile neuronal ceroid lipofuscinosis (LINCL) or CLN2 (neuronal ceroid lipofuscinosis, type 2) disease.

Classic late infantile neuronal ceroid lipofuscinosis is characterized as a disease that typically appears between age two to four years, usually and presenting with epilepsy. The seizures associated with LINCL include myoclonic, tonic, atypical absence and tonic-clonic. Following the onset of seizures, previously acquired motor/language and cognitive skills are lost. Ataxia, myoclonus and spastic quadriparesis then become prominent. Visual impairment appears but may be less obvious than in other forms of NCL. It appears between age four and six years and rapidly progresses to blindness. Affected children are usually bedridden by age six years; disabilities are severe and nursing care needs are considerable by mid-childhood. Life expectancy is between age six years and adolescence but can be longer. ${ }^{3,6,7}$ There are reported variants of LINCL caused by specific genes which in general present after 4 years of age with visual loss and seizures as the initial signs. ${ }^{3}$ Our patient showed the typical onset and course of the disease similar to what is commonly observed in the classical type of late infantile neuronal ceroid lipofuscinosis.

The EEG and MRI findings of our patient were also in accordance with what have been reported in the literature for patients with LINCL. Electroencephalographic studies among these children usually show irregular and slow background activity, accompanied with focal or generalized spike wave complexes and polyspike waves synchronous with myoclonic jerks. ${ }^{7}$ Early electroencephalogram (EEG) may show spikes in the occipital region in response to photic stimulation at $1-2 \mathrm{~Hz}{ }^{3}$ MRI frequently shows progressive cerebellar and cerebral atrophy with normal basal ganglia and thalami. ${ }^{2,3,7}$

Despite the characteristic clinical features and brain imaging findings seen in this type of NCL, CLN2/LINCL is rarely suspected or diagnosed at initial presentation to the clinic unless there is already a known or affected family member. Typically, the diagnosis only occurs after a series of misdiagnoses as the early disease course is clinically similar to many other seizure and /or metabolic disorders. To avoid delays in diagnosis, best laboratory practices have been recommended for children suspected to have the disease. ${ }^{8}$ Children who are highly suspected to have CLN2 based on clinical presentation should undergo Tripeptidyl peptidase 1 (TPP1) enzyme assay and TPP1/ CLN2 molecular test. Leukocytes isolated from whole blood are the recommended sample type for the analysis of TPP1 enzyme activity, enabling accurate and rapid diagnostic analysis. Although it is ideal that our patient should have undergone additional molecular testing for TPP1/CLN2 disease, deficient TPP1 activity in leukocytes is considered diagnostic for the disorder if consistent with the clinical presentation. ${ }^{8}$

Neuronal ceroid lipofuscinosis type 2 results from a deficient activity of the lysosomal exopeptidase Tripeptidyl peptidase 1 (TPP1) caused by mutations in the TPP1/CLN2 gene. The protease functions in the lysosome to cleave Nterminal tripeptides from substrates, and mutations in this gene is associated with the failure to degrade specific neuropeptides including a subunit of ATP synthase in the 
lysosome leading to intralysosomal accumulation of autoflourescent storage material and neuronal loss. ${ }^{3}$ It is synthesized as an inactive proenzyme (pro-TPP1) that is proteolytically processed into the active enzyme after exposure to low $\mathrm{pH}$ in vitro or targeting to the lysosome in vivo. Transfection experiments have indicated that TPP1 mutants have delayed processing and are retained in the ER rather than transported to lysosomes. ${ }^{9}$ Late infantile CLN2 disease has been lately associated with defective macroautophagosome maturation with defective endosomal/lysosomal cell processes. There was also an observed accumulation of higher amounts of Radical Oxygen Species (ROS) in late infantile CLN2 disease fibroblasts and a decrease in catalase activity could both increase the accumulation rate of peroxidized lipids and lipofuscin in late infantile CLN2 disease. ${ }^{10}$ TPP1 has also been proposed to play a central role in $\mathrm{TNF} \alpha$-induced apoptosis. ${ }^{11}$ Thus, the involvement of TPP1 in macroautophagy and TNF $\alpha$-induced apoptosis indicates that this lysosomal serine protease affects cell viability. It is still unknown, however, how TPP1-deficiency causes selective neurodegeneration. ${ }^{9}$

Although advances have been made in the genetic diagnosis for NCL, comprehensive treatment programs that delay or halt the disease progression have been elusive. Current disease management for all types of NCL is primarily targeted at controlling symptoms rather than "curing" the disease. But there are many new therapeutic possibilities that are in the pipeline for NCL. There are a number of ongoing studies in various stages of clinical trial including gene therapy, enzyme replacement therapy, stem cell therapy, small molecule carriers and RNA modulation. ${ }^{12}$ As these various treatment options are being explored, a strong foundation on which to successfully accelerate these treatments should be ensured. Necessary tools such as natural history studies, clinical rating scales, systems for early diagnosis and reliable biomarkers for tracking the disease must be highly considered as these therapeutic options are being pursued. ${ }^{12}$

In summary, our patient's severe progressive clinical course is consistent with the natural history of patients with LINCL. The cluster of characteristic clinical findings as well as their chronologic evolution may help increase the awareness of local physicians and neurologists in the identification of patients affected by this rare disease. The presence of clinical features such as seizures, ataxia and visual loss progressing to neurodegeneration should make one consider NCL. Early diagnosis is essential in order to optimize clinical care, plan for disease surveillance that will mitigate further disability, and provide genetic counselling to affected families.

\section{Statement of Authorship \\ Both authors have approved the final version of the manuscript.}

\section{Author Disclosure}

Both authors have declared no conflicts of interest.

\section{Funding Source}

None.

\section{References}

1. Nita DA, Mole SE, Minassian BA. Neuronal ceroid lipofuscinosis. Epileptic Disord. 2016; 18(S2):73-88.

2. Jadav RH, Sinha S, Yasha TC, et al. Clinical, electrophysiological, imaging, and ultrastructural description in 68 patients with neuronal ceroid lipofuscinoses and its subtypes. Pediatr Neurol. 2014; 50(1):85-95.

3. Mole SE, Williams RE. Neuronal ceroid lipofuscinosis. [Online]. 2013. [cited 2017 Feb]. Available from https://www.ncbi.nlm.nih.gov/ books/NBK1428/.

4. Warrier V, Vieira M, Mole SE. Genetic basis and phenotypic correlations of the neuronal ceroid lipofuscinoses. Biochim Biophys Acta. 2013; 1832(11):1827-30.

5. Cooper JD, Tarczyluk MA, Nelvagel HR. Towards a new understanding of NCL pathogenesis. Biochim Biophys Acta. 2015; 1852(10 Pt B):225661.

6. Mink JW, Augustine EF, Adams HR, Marshall FJ, Kwon JM. Classification and natural history of the neuronal ceroid lipofuscinosis. J Child Neurol. 2013; 28(9):1101-5.

7. Chang X, Huang $Y$, Meng $H$, et al. Clinical study in Chinese patients with late infantile form neuronal ceroid lipofuscinosis. Brain Dev. 2012; 34(9):739-45.

8. Fietz M, Al Sayed M, Burke D, et al. Diagnosis of neuronal ceroid lipofuscinosis type 2 (CLN2 disease): Expert recommendations for early detection and laboratory diagnosis. Mol Genet Metab. 2016; 119(12):160-7.

9. Cárcel-Trullols J, Kovács AD, Pearce DA. Cell biology of the NCL proteins: what they do and don't do. Biochim Biophys Acta. 2015; 1852(10 Pt B):2242-55.

10. Vidal-Donet JM, Cárcel-Trullols J, Casanova B, Aguado C, Knecht E. Alterations in ROS activity and lysosomal $\mathrm{pH}$ account for distinct patterns of macroautophagy in LINCL and JNCL fibroblasts. PLoS One. 2013; 8(2):e55526.

11. Autefage H, Albinet V, Garcia V, et al. Lysosomal serine protease CLN2 regulates tumor necrosis factor-alpha-mediated apoptosis in a Biddependent manner. J Biol Chem.2009; 284(17):11507-16.

12. Geraets RD, Koh SY, Hastings ML: Kielian T, Pearce DA, Weimer JM. Moving towards effective therapeutic strategies for neuronal ceroid lipofuscinosis. Orphanet J Rare Dis. 2016; 11:40. 